\title{
Visualization Enables Accomplishment A Systems Response to Global Calls for Reduction of Harm to Patients in Healthcare
}

\author{
Ranjit Singh \\ MBBChir MA (Cantab.) MBA \\ Member of WHO Expert Panel for Patient Safety in Primary Care. \\ Vice-Chair for Research, Department of Family Medicine, Director UB Patient Safety Research Center, \\ Associate Professor, Schools ofMedicineand Management, State University of NY at Buffalo, NY USA, \\ rs10@buffalo.edu
}

\begin{abstract}
The author and his team's mission is to reduce the burden of harm to patients all over the world. The economic impact of harm to patients in US alone is more than $\$ 1$ trillion/year, out of a total annual healthcare cost of \$3.2 trillion. The World Health Organization regards patient safety a Basic Human Right. The business case for harm reduction is strong. This business should be carried out in a common universal language that is visual. World needs a bottom-up prospective approach to harm reduction because the currently prevalent retrospective methods lead to dissatisfaction in all stakeholders. Such an approach has been developed and successfully used by the author to reduce frequency and severity of harm. Background to the problem and its solution are presented.
\end{abstract}

Keywords-cost; errors; harm; healthcare; patient; quality; safety; visualization.

\section{INTRODUCTION}

Efforts for reducing the huge global burden of patient harm in healthcare should take advantage of recommendations in two recent reports: (1) The September 2012 IOM Report ${ }^{[1]}$ thatcalls for higher quality at lower cost by fostering continuous learning. IOM very rightly asserts that there can be no quality without safety, (2) August 2013 report $^{[2]}$ to the British Prime Minister by National Advisory Group on the Safety of Patients in England, calls for: "A promise to Learn - A Commitment to Act: Improving the Safety of Patients in England". Both reports make the vital statement that a bottom-up approach to quality improvement is much more powerful than top-down efforts that are based on rules, standards and enforcement.

The economic impact of harm to patients in US is more than $\$ 1$ trillion/year, out of a total annual healthcare cost of \$3.2 trillion. The World Health Organization regards patient safety a Basic Human Right. Reduction of avoidable scale of harm and costs should receive priority from all stakeholders. Despite this the literature on this avoidable healthcare outcome is relatively scarce ${ }^{[3]}$. Low and middle-income nations face a much larger challenge. In 1974, Webster stated: "Safety is Good Business". A safe organization is a cost-effective quality organization. The business case for harm reduction is strong. ${ }^{[4]}$

\section{VALUE OF VISUALIZATION}

As a well-respected author and speaker, Stephen Covey put it, "Visualizing something organizes one's ability to accomplish it". The author and his team's mission is to reduce the burden of harm to patients all over the world. Visualization can help tostructure and illustrate the 'story' of an error or event. It is a universal tool that furnishes anatural common 'language'. It providesa fast path to engaging minds of individualsand teams, insight into causes, cascades and consequences oferrors (with the potential forimproved outcomes),aid for coping with the complexities, fragmentation and decentralization of the healthcare system, andaid for mapping across different taxonomies and data structures. ${ }^{[5]}$ Applying a systems engineering/management approach,the author has developed visual models at macro and micro levelsshown in Figures I and II.These have been successfully used to reduce harm.

\section{A. Micro Level Visualization}

Micro-level models are close-up views; each may represent one or more points within the healthcare system for a specific domain, or for a specific process within a domain. These models show how the various entities/agents in the microsystem interact. The level of detail represented in these depends on the purpose of its use. Figure I is an example for medication management in outpatient setting and shows activities in the office, pharmacy, home, laboratory, imaging/radiology facility, third party payer, and interactions within and between these. Errors or safety problems can originate at any, or at multiple points within the system.

\section{B. Macro Level Visualization}

This is a high-level view (Figure II) of the healthcare system. The processes of care are represented by the radials. These processes are recognized to occur in a cyclical fashion as show by the clockwise progression from Assessment to Plan to Implementation, Feedback, Review and Learn and back to Assessment again. The innermost circle represents the patient in his/her own domain and recognizes that this is the place where most 'health-care' actually occurs. Office-based primary care is represented by circle No.1. The main purpose of this macro-system model is to understand a patient's care in the context of the overall healthcare system, especially with respect to errors and opportunities for errors, including in transitions between different parts of the system. This helps to understand interdependencies and needs for information flow. 


\section{THE THREE B'S OF HEALTHCARE}

Better Health, Better Healthcare and Better Value" (3Bs)aresocietal, ethical, and fiscal imperatives. In the US "Partnership for Patients: Better Care Low Costs" HHS initiatives are designed to shift control and power from caregivers to patients, to make care "patient-centered. ${ }^{[6]}$ The needs expressed in the Patient Protection and Affordable Care Act must be addressed systematically by developing and assessing innovative methods which include patientengagement. Because one size does not fit all, these methods should be driven by local teams and should be easily adaptable to all healthcare settings, each of which is unique. This is essentially a bottom-up engagement approach that is synergistic with skillful non-hierarchical leadership. The management methods should encourage system-based practice and continuing practice-based learning and harm reduction in a transparent environment. Figure III presents the Systemic Visualization of the Global Mission for minimum harm Healthcare. It interrelates the Global mission, Objective of creating high reliability organization for minimum harm, Domains ofhealthcare and Income groups. Figure IV (on last page) draws attention to some essential contributors to fulfilling the requirements of the US Patient Protection and Affordable Care Act.The three B's are described below.

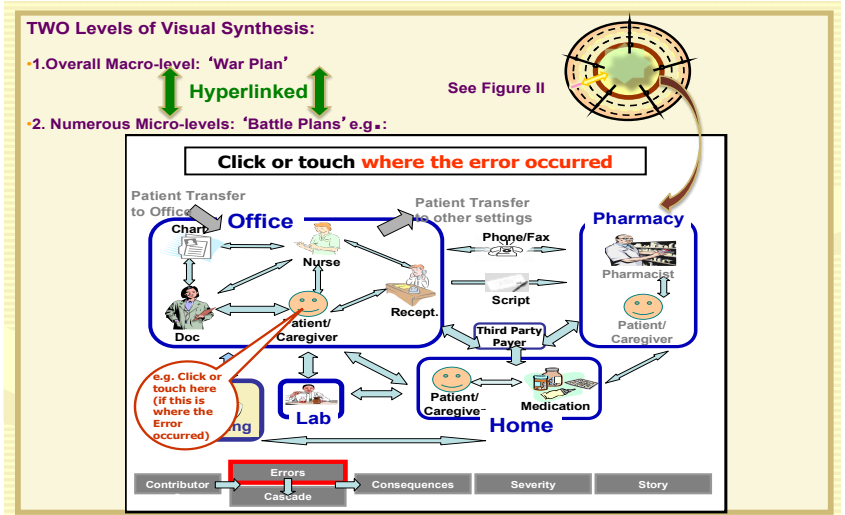

FIGURE I: VISUALIZATION INTERLINKED MACRO AND MICRO LEVELS.

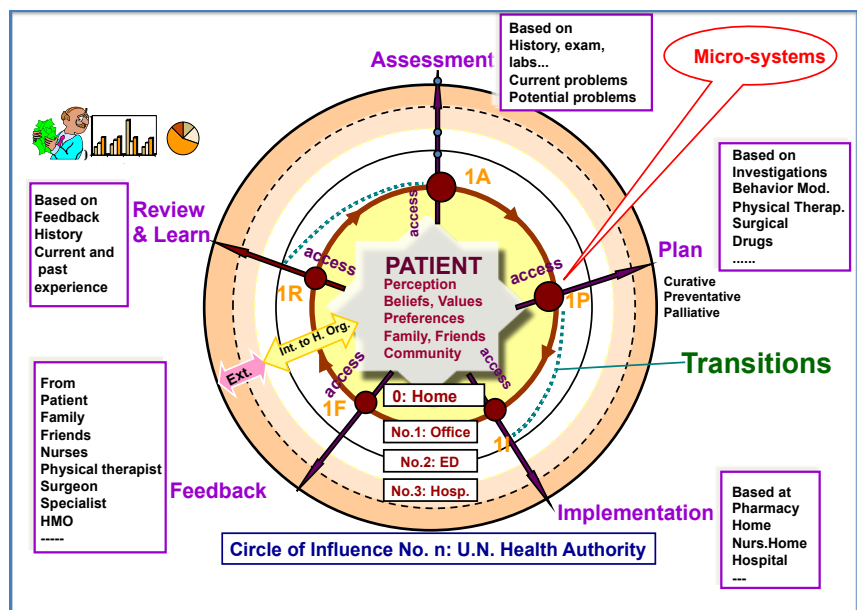

FIGURE II. DETAILED VISUALIZATION AT MACRO LEVEL

\section{A. First B of Healthcare: Better Health}

The contributors to better health, for individuals and their community, shown on left side of Figure IV, include good:(a) affordableaccess to healthcare, (b) education - especially health literacy, (c) healthy food, (d) jobs and employment, (e) social services, (f)public safety, (g) social and family networks that are female based, (h) playgrounds, and (i) safe streets.

\section{B. Second B of Healthcare: Better Care}

For harm reduction stakeholders have to be armed with sharp awareness of and attention to the following systemic threats: complexity of the process of care, variability from patient to patient, inconsistency in the standards of care, poorinterfacing (e.g. transition between settings), lack of errorpreventing barriers,lack of initiative to handle the unforeseen, use of inappropriate time constraints, use of hierarchical culture in the system, and human fallibility - to Err is Human. Section IVbriefly presents the approaches to harm.

\section{Third B of Healthcare: Better Value}

As stated earlier Webster stated: "Safety is Good Business".Harm reduction approaches need to be based on prioritization of various hazards. Relationship between Costs and Harm reduction is portrayed on the right hand side of Figure IV.As can be expected rate of cost reduction occurs at a decreasing rate with continuing reduction in harm. This figure draws attention to the prime contributors to cost reduction.

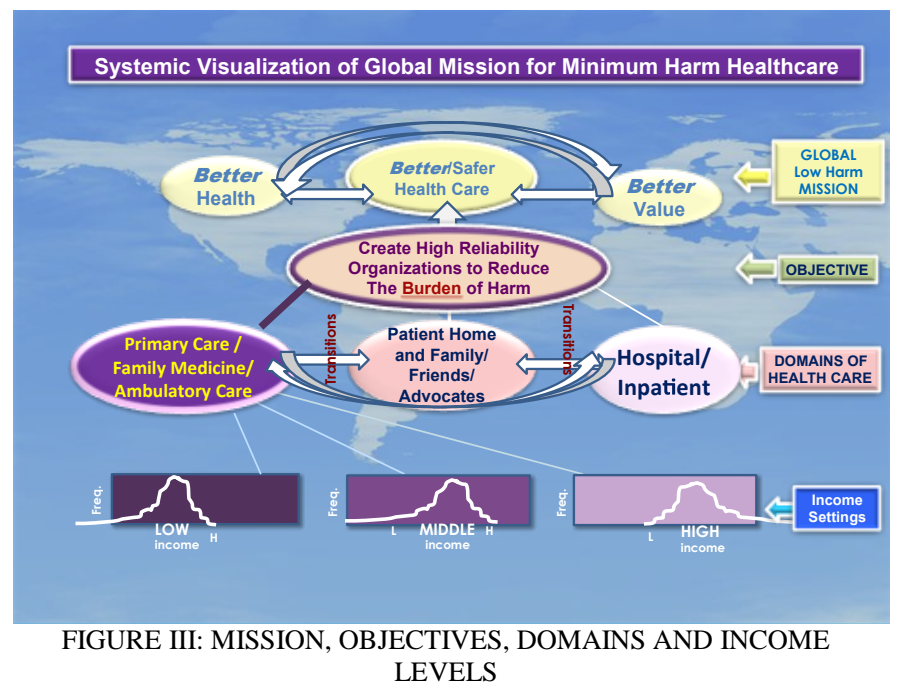

\section{METHODOLOGIES FOR HARM REDUCTION}

Two basic approaches arecurrently used to estimate the burden harm. These are retrospective and prospective.Theycan be complementary. Figure $\mathrm{V}$ portrays these.

Retrospective: Shown on left side, error reports, internal and external audits, quality and safety indicators, and trigger tools are some of these. Each reveals only a tip of iceberg of safety gap and somewhat different perspectives of the same reality. Generalizations of the results from retrospective methods lead to stakeholders' dissatisfaction. These methods tend to be top-down and do not fully meet needs expressed earlier. The US Inspector General of HHS has recently stated: "Current methods of detection of Adverse Events are inadequate" and riskmisdirection of present efforts to improve 
safety based quality.Better methods are called for. ${ }^{[7]}$ Error reporting often does not promote understanding of the organizational structure and processes of care. Instead, it tends to be associated with blame and shame and frequently results in antagonism among team members, undermining mutual respect, trust, and cooperation.Sadly, these inadequate methods are the ones currently used in research to compare the efficacy of safety improvement interventions. We need a change in mind set in our approaches to safety measurement and monitoring.

Prospective: The aim of all safety efforts must be to avoid potential harm. This approach, shown on right side of Figure $\mathrm{V}$,captures the memory ${ }^{[8]}$ of all stakeholders in any setting, tries to anticipate safety events in contrast to retrospective methods that only respond after an event. The root methodology in this category is failure modes and effects analysis (FMEA). This leading indicator has been widely used in other high-risk industries to identify weaknesses (failure modes) and possible consequences of failure (effects) and to prioritize areas for improvement. The Joint Commission has required, since 2002, that all accredited hospitals perform a proactive risk assessment each year following a series of steps based on FMEA ${ }^{[9,10]}$ This process, usually made up of eight steps, is time consuming, costly, and requires considerable expertise and experience.

Prospective methods are effective because they can foster an active and transparent engagement of patients and providers at the point of patient care.These invoke the paradigm of complex adaptive systems calling for each health care setting to be viewed as a complex microsystem. These settings have to be adaptive to survive and thrive. These are endowed with simple rules, shared vision, and opportunities for team members to innovate That is to say, they: (1) generate information about the vulnerabilities of their microsystem and promote dissemination of this information to aid learning about these vulnerabilities (the hallmarks of safety culture, as pointed out by the National United States National Patient Safety Foundation); (2) pool diverse resources to generate options for responding to unpredictable external and internal stimuli/pressures; and (3) create good teams to perform tasks 1 and 2 successfully and keep the prevailing anxieties in check.

In light of the above the author and his team have appropriately adapted FMEA method. This visual method, termed Systematic Appraisal of Risk and Its Management for Error Reduction (SARAIMER), can be cost effectively used in all settings to reduce harm with bottom-up optimized interventions as demonstrated by Singh et all. ${ }^{[9,10]}$ (with support from AHRQ grants). AHRQ has recently published "A Toolkit for Rapid-Cycle Patient Safety and Quality Improvement" that incorporates this SARAIMER approach. ${ }^{[1]}$

\section{CONCLUDING REMARKS}

SARAIMER is intended to form self-empowered learning teams motivated to prioritize safety problems and to devise feasible interventions, thereby fostering site specific [12] sustainable culture of safety. The August 2013 report by National Advisory Group on the Safety of Patient in England "A promise to learn- a commitment to act" ${ }^{[2]}$ synergizes with the core of the methodology put forward by the author. Hisobjective is to disseminate this highly transferable bottomup approachas widely as possible.

\section{ACKNOWLEDGEMENT}

The author is grateful to AHRQ for research grant and its support. Work by all the team members is gratefully acknowledged.

\section{REFERENCES}

[1] IOM. Best Care at Lower Cost: The path to continuously Learning Health Care in America. September 2012.

[2] National Advisory Group on the Safety of Patients in England. A promise to learn - a commitment to act. August 2013. National Health Service, UK.

[3] B. Balik and F. Dobkiss. 10 Years After To Err is Human: Are we Listening to Patients and Families Yet? Focus on Patient Safety Newsletter.2010;13(1):1-3. http://www.npsf.org/paf/npsfp/fo/pdf/Focus_vol_13_1_2010.pdf.

[4] P.K. Lindenauer. Strengthening the Business Case for Patient Safety. May 2013. AHRQ Perspectives Safety.http://webmm.ahrq.gov/perspective.aspx?perspectiveID $=142$

[5] R.Singh, A. Singh, et al Creating a common vision for all stakeholders to make healthcare safer with interactive visual monitoring. International Journal of Advanced Intelligence Paradigms.2011;3(1).

[6] C.R. Denham. The Partnership With Patients: A Call to Action for Leaders. Patient Saf, Volume 7, Number 3, September 2011.

[7] D.R. Levinson. Adverse Events in Hospitals: National Incidence Among Beneficiaries: Department of Health and Human Services: Office of Inspector General.; November 2010/2011.

[8] L. Donaldson. An organisation with a memory. Stationery Office, Jan 1, 2000 - Great Britain.

[9] R. Singh, D.R. Anderson, E.A. McLean-Plunkett, R. Brooks, A. Wisniewski, and G. Singh. IT-Enabled Systems Engineering Approach to Monitoring and Reducing ADEs. Am J Manag Care 2012;18(3):169175.

[10] R.Singh, D.R. Anderson, E.A. McLean-Plunkett, A. Wisniewski, R. Kee, C. Fox, G. Singh.Effects of Self-Empowered Teams on Rates of Adverse Drug Events in Primary Care.Int J Fam Med vol. 2012, Article ID 374639, 6 pages, 2012.

[11] AHRQ. Improving Your Office Testing Process: A Toolkit for RapidCycle Patient Safety and Quality Improvement. Rockville, MD: Agency for Healthcare Research and Quality; August 2013.

[12] R. Singh, D. Anderson, E. McLean-Plunkett, D. Raheja, G. Singh. Interplay between Quantifiable andUnquantifiable Safety Climate as Affected by Successful Systems Approach to Medication Safety Improvement: Primary Care Settings. American Journal of Medicine and $\begin{array}{llll}\text { Medical } & \text { Sciences } & \text { 2013, } & \text { 3(1): }\end{array}$ 


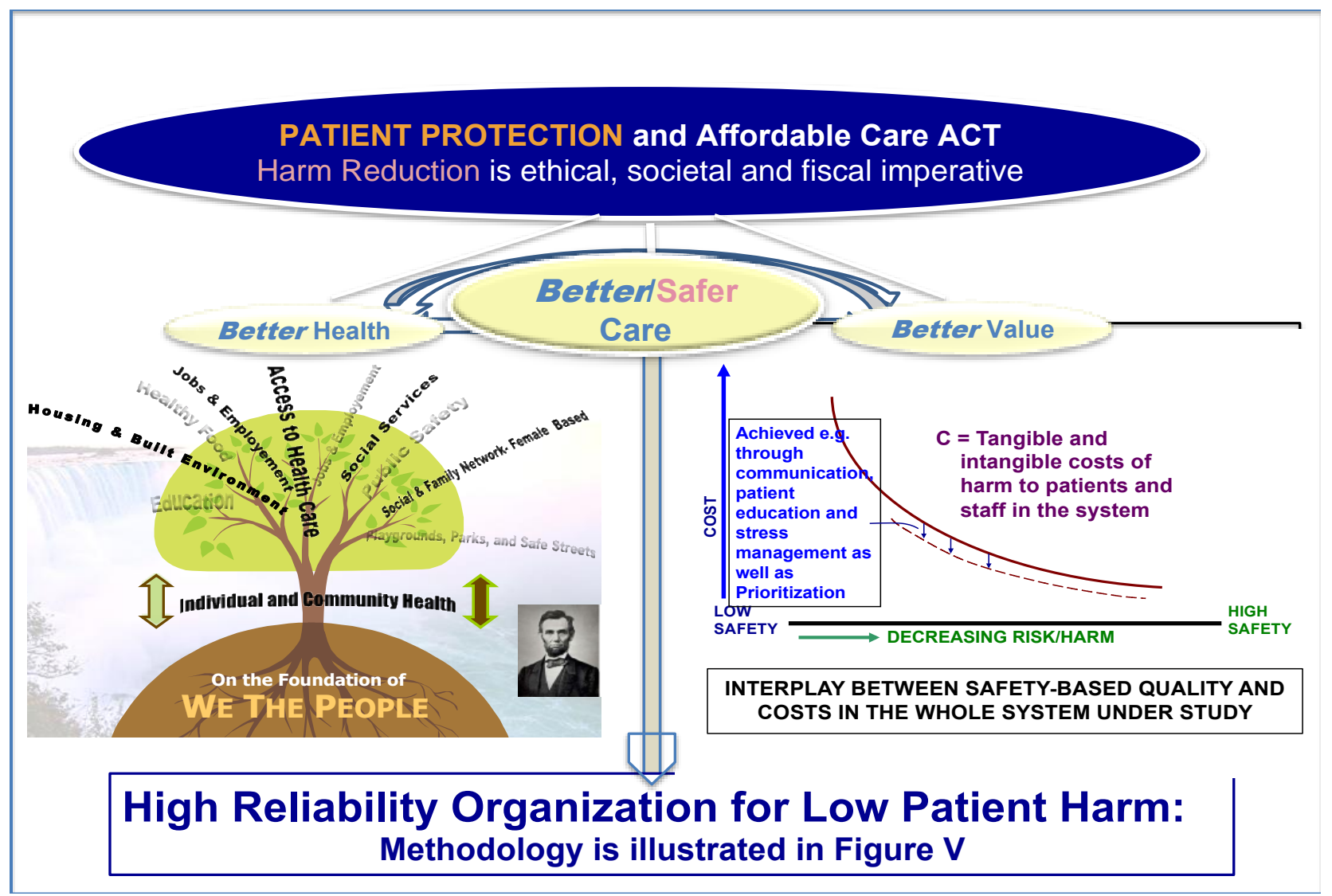

FIGURE IV: THREE CONTRIBUTORS TO MEETING THE NEEDS OF PATIENT PROTECTION AND AFFORDABLE CARE

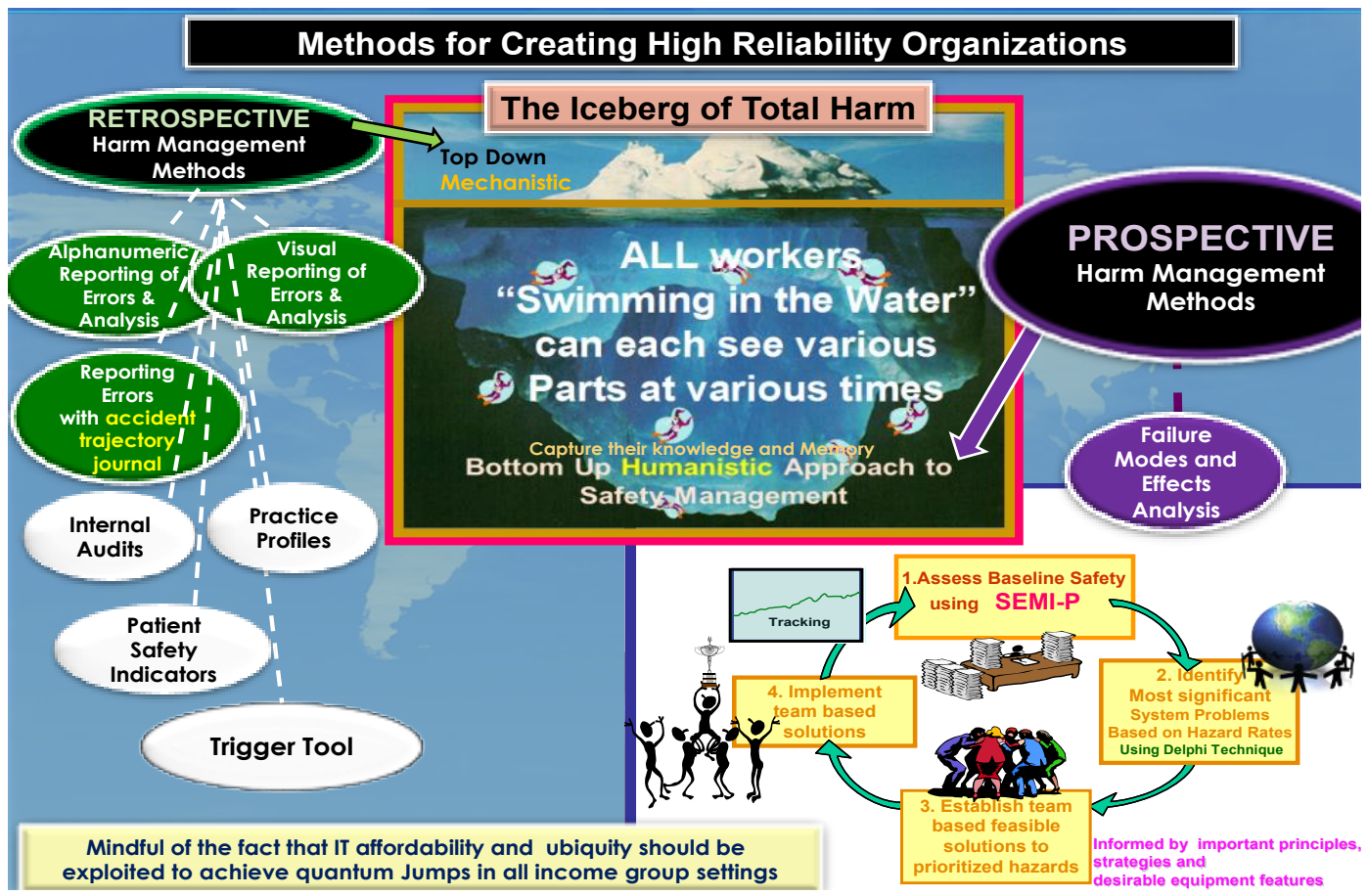

FIGURE V: RETROSPECTIVE AND PROSPECTIVE APPROACHES TO HARM ASSESSMENT AND ITS REDUCTION 